\title{
Taste and emotionality in rats selectively bred for high versus low saccharin intake
}

\author{
NANCY K. DESS \\ Occidental College, Los Angeles, California \\ and \\ THOMAS R. MINOR \\ University of California, Los Angeles, Los Angeles, California
}

\begin{abstract}
Rats were selectively bred for high versus low saccharin ingestion, a putative measure of enhanced stress and emotionality (Dess, 1991). In Experiment 1, third-generation Occidental highsaccharin (HiS) and low-saccharin (LoS) rats were tested for saccharin ingestion and emotionality. The saccharin test confirmed that the lines differed on the selection phenotype. In addition, LoS rats were more emotional, as evidenced by longer emergence latencies and more defecation in a modified open-field test. In Experiment 2, LoS rats had lower quinine preference scores and drank saccharin-adulterated glucose less avidly. These outcomes are reminiscent of the behavior shown by inescapably shocked rats. Unlike helpless rats, however, LoS rats drank less avidly during a dilute sucrose test, an effect more reminiscent of chronic mild stress. The lines did not differ reliably on intake of concentrated glucose or Polycose, even when the latter was mixed with saccharin. In Experiment 3 , LoS rats preferred saccharin less strongly than did HiS rats at concentrations of $0.05 \%$ to $0.7 \%$ and had an aversion to a $1.0 \%$ solution. In Experiment 4, LoS rats were affected more by shock, as assessed by stress-induced anorexia. These and other recent findings support the notion of shared mechanisms for taste, emotionality, and stress vulnerability.
\end{abstract}

Ingestion interacts extensively with affective processes, such as fear and distress (Dess, 1991). For instance, exposure to intermittent electric shock alters the distribution of meals and eating rate in a manner suggestive of a persistent state of fear or anxiety (Dess \& VanderWeele, 1994; Helmstetter \& Fanselow, 1993). Experience with inescapable tailshock also increases "finickiness" (Jacobs \& Sharma, 1969; Sclafani, Springer, \& Kluge, 1976): Relative to nonshocked controls, shocked rats consume less of quinine- or saccharin-adulterated substances but increase their intake of sugar (Dess \& Chapman, 1990; Dess, Chapman, \& Minor, 1988; Dess \& Choe, 1994; Dess, Minor, \& Brewer, 1989). Thus, stress changes when, how, and what rats consume (see also Katz, 1982; Plaznik, Stefanski, \& Kostowski, 1989; Vaswani, Tejwani, \& Mousa, 1983; Willner, Towell, Sampson, Sophokleous, \& Muskat, 1987).

Stress-induced finickiness varies among rats, and the expression of these individual differences varies with

This research was supported by NIMH Grant MH41107 to T.R.M. and N.K.D., by a grant from the Pew Charitable Trust to N.K.D. and W. S. Agras, and by the Dean of Faculty at Occidental College. The authors would like to thank Kimberly Black, Steve Chang, Seung Choe, and Heather Furner for their assistance in collecting the data. Correspondence should be addressed to N. K. Dess, Department of Psychology, Occidental College, 1600 Campus Rd., Los Angeles, CA 90041 (e-mail: dessnk@oxy.edu).

-Accepted by previous editor, Vincent M. LoLordo stressor severity (Minor, Dess, Ben-David, \& Chang, 1994). Moreover, rats with low prestress body weights are more finicky than are heavier rats following exposure to escapable shock (Dess \& Chapman, 1990). These data suggest the mediating action of some constitutional variable, the nature and origin of which has not been determined. Identifying such variables is difficult with subjects obtained from random stock which can differ in age, parentage, life history, and so forth.

Selective breeding is a powerful way to examine individual differences in taste and their relationship to affective processes. Differential saccharin intake has been selectively bred in rats (Nachman, 1959). Virtually nothing is known, however, about how selecting rats on the basis of saccharin intake alters behavior toward other tastants. Saccharin consumption correlates positively with ethanol consumption among outbred rats (Gosnell \& Krahn, 1992). Similarly, rats bred for high ethanol consumption drink more saccharin solution (Overstreet et al., 1993), although results for other tastants vary among strains (Sinclair, Kampov-Polevoy, Stewart, \& Li, 1992). Although ethanol is interesting in its own right, its complex concentrationdependent taste, caloric, and pharmacological properties complicate interpretation of the saccharin-ethanol correlation. One goal of the present study was to use more and simpler flavors to help determine the role of various taste qualities in differential saccharin intake.

A related area of research is concerned with noningestive correlates of saccharin intake. Rats selectively bred on an emotionality phenotype (Gray, 1987) also dif- 
fer in saccharin consumption (Overstreet et al., 1993) and stress-induced suppression of quinine intake (Brush et al., 1988). The more emotionally reactive lines drink less saccharin and less quinine. Whether breeding on a saccharin phenotype yields lines that will differ in emotionality is not known.

The present paper presents data from a selective breeding project which was initiated upon the incidental discovery of a male rat that was averse to saccharin solution. This male and an avid saccharin-drinking male were mated with different pairs of females with unremarkable saccharin preference to create the Occidental LowSaccharin (LoS) and High-Saccharin (HiS) lines. Samples of first- and second-generation LoS and HiS male progeny showed no difference in saccharin intake. However, first-generation rats did differ in their aversion to a weak quinine solution (quinine preference score: $\operatorname{LoS}=$ $0.26 \pm 0.09$; HiS $=0.43 \pm 0.04$ ). These preliminary data suggested that the fathers responded differently to saccharin's bitter component or other aversive taste properties. In open-field testing, second-generation LoS males took longer to emerge into a novel field (mean $=207.0 \pm$ $27.0 \mathrm{sec}$ ) than did HiS males (mean $=73.5 \pm 21.0 \mathrm{sec}$ ).

In the research presented here, we were concerned with whether the HiS and LoS lines differed with respect to their intake of saccharin and other flavors, and whether any such differences correlated with emotionality and stress vulnerability (Dess, 1991). In Experiment 1, to assess line separation, LoS and HiS rats were observed in a test with $0.1 \%$ saccharin and tested for emotionality in a novel open field. In Experiments 2 and 3, respectively, the lines were also tested for their reactions to several tastants and saccharin concentrations. Finally, in Experiment 4, the relative impact of inescapable shock on eating, drinking, and body weight in the HiS and LoS lines were assessed.

\section{EXPERIMENT 1}

In Experiment 1, selectively bred rats were tested for intake of and preference for $0.1 \%$ saccharin and for potential emotionality correlates. Emotionality was assessed in a novel open field. Behavior in an open field has been used to measure fearfulness or emotionality (Walsh \& Cummins, 1976). Of the many behaviors observable in the open field, emergence and movement latency and defecation are reasonably valid (Royce, 1977). If individual differences in saccharin intake and fearfulness are robustly related (cf. Minor et al., 1994), LoS rats should be more fearful in a well-lit novel open field.

\section{Method}

Subjects. The HiS and LoS lines were derived from Holtzman stock (HSD Inc., Indianapolis). The lines were developed by breeding rats with extreme scores on drinking during 24-h access to saccharin and water. Specifically, saccharin intake is expressed as change from a water-only baseline, which then is transformed to a percentage of body weight. The highest scorers in the HiS line and the lowest scorers in the LoS line were used for breeding. Rats with an extreme water baseline relative to littermates were excluded from the breeding pool. Half of the males used to produce the second generation were selected from rats obtained from HSD Inc. to expand the pool of genetic and other constitutional variables.

Thirty HiS rats ( 13 males and 17 females) and 30 LoS rats (16 males and 14 females) from two litters of the third generation in each line were used. The litters were well matched for litter size, sex ratio, and age. At 30 days of age, the rats were weaned and group-housed with same-sex littermates. During rearing and testing, the rats were maintained on a 12:12-h light:dark cycle, with light onset at 7:00 a.m. PST. Purina 5001 Chow and tap water were freely available throughout the experiment.

Apparatus. The open field consisted of an enclosed startbox $(25 \times 8 \times 18 \mathrm{~cm})$, which opened near a corner of a square field $(56 \times 56 \times 100 \mathrm{~cm})$. The apparatus was made of black acrylic except for the clear acrylic floor. Black tape on the underside of the field $15 \mathrm{~cm}$ from the walls bisected each side and defined periphery and center. The startbox was dark, and the field was illuminated by a $40-W$ frosted bulb located directly above the arena. A small plastic ball was placed in the center of the field at the beginning of each test. A few food pellets (Formula A, P. J. Noyes Co., Lancaster, NH) were placed in each of two small plastic cups located outside the startbox. Additional pellets were placed on the floor at the center of each of the side walls and in each of the corners.

The saccharin solution was $0.1 \%(\mathrm{wt} / \mathrm{vol})$ sodium saccharin (Fisher Scientific, Pittsburgh) made daily with tap water. Fluids were presented in $50-\mathrm{ml}$ polypropylene tubes or $250-\mathrm{ml}$ glass bottles with a rubber stopper and stainless steel spout. When only water was available, both a tube of water and a bottle of water were used. When water and saccharin were available, the tube contained water and the bottle contained saccharin

Procedure. Open-field testing occurred at 65-80 days of age. Same-sex HiS and LoS rats were tested in each session. The rats were moved in their group cages to a darkened room outside the testing room, where they were left undisturbed for $1 \mathrm{~h}$ before testing.

A test began with placement of a rat in the startbox. After approximately $20 \mathrm{sec}$, the door to the field was opened. Head-out (ears across threshold), front-out (front paws and shoulders across threshold), and full emergence (base of tail across threshold) latencies were recorded. Upon full emergence, the door to the startbox was lowered. Behaviors counted in the field included peripheral line crossings, entrances to the center, and grooming bouts. After $5 \mathrm{~min}$, the rat was returned to its group cage; a maximum latency of $300 \mathrm{sec}$ was recorded for failure to partially or fully emerge. Number of pellets eaten and fecal boli were counted. Missing pellets were replaced, and the floor of the apparatus was sponged and toweled dry. Rats from the two lines were alternated. After all rats had been tested, they were returned to group home cages in the vivarium.

The open-field test was repeated the next day. After all rats finished the 5-min emergence test, each rat was placed directly into the field near the food cups and was observed for $2 \mathrm{~min}$; the door to the startbox was closed. Peripheral line crossings, entrances to the center, grooming, eating, and defecation were recorded.

Following the open-field test, the rats were individually housed. Saccharin testing began approximately 2 weeks later. Average daily intake of tap water over a 48-h period was used as a baseline. The rats were weighed on the second baseline day. For the next $48 \mathrm{~h}$, saccharin solution and water were continuously available, except for approximately $30 \mathrm{~min}$ midway through the test when the bottles were weighed and refilled. The left-right positions of saccharin and water were balanced across rats and days.

Statistical analyses. In this and subsequent experiments, baseline water intake and body weight were compared using line $X$ sex analyses of variance (ANOVAs). When a baseline measure differed between lines, it was included as a covariate in a preliminary analysis of test data (i.e., ANCOVA). When body weight was the covariate, within-sex $z$ scores were used to avoid the confounding of 
sex with other influences on body weight. The covariates were tested for significance at $\alpha=.10$ to determine the potential impact of baseline differences on test behavior.

In the two instances of a significant baseline covariate (for quinine preference in Experiment 2 and for saccharin avidity and weight gain in Experiment 4), ANCOVA results are reported in the text and adjusted means are shown in the figures. Otherwise, results from ANOVAs performed without the covariate are reported and corresponding raw group means are shown in the figures. In every data set, the ordering of adjusted group means was identical to the ordering of raw means, and the exclusion of nonsignificant covariates in the simpler ANOVAs had no effect on the significance of any line or sex effect at $\alpha=.05$.

\section{Results}

Baseline water intake did not differ between the LoS and HiS lines. LoS rats were heavier than their HiS counterparts $[F(1,56)=9.16]$, although the difference was due mostly to the females (LoS mean $=318.8 \pm 5.9 \mathrm{~g}$; HiS mean $=281.4 \pm 5.1 \mathrm{~g})$ rather than to the males (LoS mean $=488.4 \pm 12.0 \mathrm{~g}$; HiS mean $=474.8 \pm 8.5 \mathrm{~g}$ ). When body weight was used as a covariate in analyses of the test data, no covariation was detected.

Open-field tests. Mean emergence times from the first open-field test are shown in Figure 1. LoS rats emerged more slowly than did HiS rats, and males emerged more slowly than did females. Two-way ANOVAs with line and sex as variables showed that the line effect was significant for each latency measure [for head-out, front-out, and full-emergence latency, respectively, $F \mathrm{~s}(1,56)=$ $7.36,10.65$, and 4.96$]$, as was the sex effect $[F \mathrm{~s}(1,56)=$ $10.04,18.25$, and 14.43].

Many of the rats that did not emerge into the open field during Test 1 did emerge in Test 2 . Conversely, many rats that had emerged fully during Test 1 failed to do so in Test 2. The failure to see consistent evidence of exploratory behavior in rats that had emerged in Test 1 may have been a procedural artifact. Closure of the guillotine door after a rat had left the startbox often resulted in scrabbling at the door, as if blocking of this escape route was

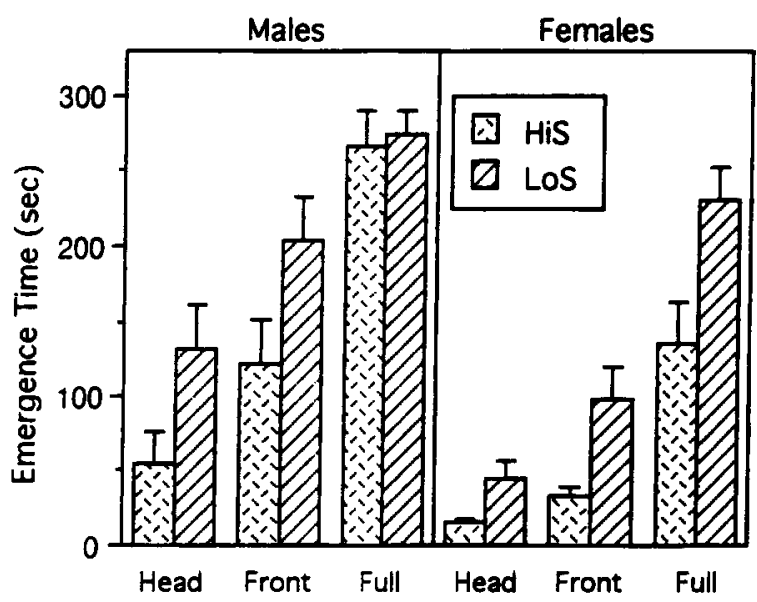

Figure 1. Experiment 1: Mean emergence latencies (head-out, front-out, and full) in a 5-min novel open-field test of male and female HiS and LoS rats.
Table 1

Experiment 1: Total Defecation During Open-Field Testing and Line Crosses in the 2-Min In-Field Test

\begin{tabular}{|c|c|c|c|c|}
\hline & \multicolumn{2}{|c|}{$\begin{array}{c}\text { Defecation } \\
\text { (Number of Boli) }\end{array}$} & \multicolumn{2}{|c|}{$\begin{array}{l}\text { Number of } \\
\text { Line Crosses }\end{array}$} \\
\hline & $M$ & $S E M$ & $M$ & $S E M$ \\
\hline HiS males & 3.3 & 0.7 & 5.5 & 1.0 \\
\hline LoS males & 7.6 & 1.3 & 5.2 & 0.9 \\
\hline HiS females & 3.9 & 1.1 & 5.2 & 0.9 \\
\hline LoS females & 2.6 & 0.9 & 6.5 & 1.2 \\
\hline
\end{tabular}

frightening. Rats that behaved in such a manner in Test 1 were less likely to explore the open field in Test 2 . Because of this complication, Test 2 emergence data were not analyzed.

Mean total defecation during the 12 min of open-field testing over the 2 test days is shown in Table 1 . LoS males defecated more than did HiS males, whereas LoS and HiS females defecated about equally often. A mixeddesign ANOVA (line $\times$ sex $\times$ placement) yielded a significant main effect of $\operatorname{sex}[F(1,56)=4$.19] and a line $X$ sex interaction $[F(1,56)=6.70]$. Post hoc ANOVAs showed that LoS and HiS males differed significantly $[F(1,27)=7.39]$, whereas LoS and HiS females did not. No effect involving repeated placement was significant, indicating that neither the different lengths of the tests $(2$ or $5 \mathrm{~min}$ ) nor door closure in the first test had much effect on defecation.

Eating, grooming, and entrances to the center of the field were rare. For example, of all rats tested, only 4 ate pellets (1 HiS male and 3 HiS females). Analyses of these measures yielded no significant effects. Mean number of line crossings during the 2-min in-field test on Day 2 (see Table 1) did not differ as a function of line or sex.

Saccharin test. Because a glass bottle and a polypropylene tube were used during the saccharin tests, potential container preferences were assessed during the water baseline period by calculating a tube (vs. glass) preference score for each rat. An analysis of these scores yielded no significant effect of line or sex. Moreover, the mean preference score did not deviate significantly from indifference $(0.50)$ in any group.

Two measures of saccharin ingestion were analyzed. An avidity score was obtained by calculating total fluid consumption on each test day and subtracting the rat's water intake during the baseline period; thus, each rat served as its own fluid-intake control. These values were transformed to a percentage of the rat's body weight to facilitate comparison of males' and females' scores. The resulting score reflects the impact of saccharin availability on total fluid consumption. A conventional saccharin preference score was also calculated by dividing saccharin intake by the total amount of fluid consumed in the test.

Mean avidity in the two 24-h saccharin tests is shown in the upper panels of Figure 2. LoS rats drank less during the saccharin tests than did HiS rats, and males drank less than did females. The line difference was numeri- 
cally larger for females than for males. Overall, drinking decreased between tests, especially among HiS rats. Saccharin generally was preferred to water (lower panels of Figure 2), but LoS rats preferred saccharin less strongly than did HiS rats.

A mixed-design ANOVA (line $X$ sex $X$ test day) on avidity scores yielded significant effects of line $[F(1,56)=$ $28.81]$, sex $[F(1,56)=22.97]$, and test day $[F(1,56)=$ $10.23]$, and significant line $\times \operatorname{sex}[F(1,56)=4.78]$ and day $\times$ line interactions $[F(1,56)=4.87]$. The same type of ANOVA on preference scores yielded only a significant line effect $[F(1,56)=5.40]$.

\section{Discussion}

Selection for differential ingestion of saccharin was successful. LoS rats drank less avidly during saccharin testing and preferred saccharin to water less strongly than did HiS rats. The LoS and HiS rats also behaved differently in the open field. LoS rats, especially males, were more emotional than HiS rats by emergence-latency and defecation measures. These measures differ in their sensitivity to general motor activity, exploratory or "ludic" (Berlyne, 1969) behavior, and autonomic tone. As such, the difference between lines probably is not limited to only one of these processes (Royce, 1977). A parsimonious explanation of the differences in latency and defecation measures and the lines' similarity on line crossing is that the LoS rats were more fearful than the HiS rats. The parallel between the open-field and saccharin data supports the prediction that selection on the saccharin phenotype should yield noningestive correlates with a strong affective component (see Dess, 1991, 1993).

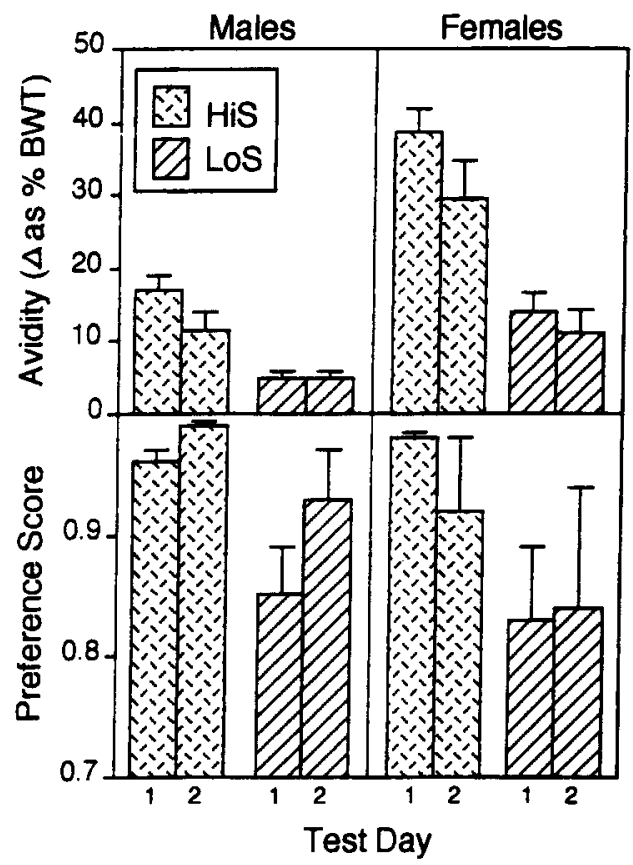

Figure 2. Experiment 1: Results of two 24-h two-bottle tests for saccharin $(0.1 \%)$ avidity $-[($ total $\mathrm{ml}-$ water $\mathrm{ml}) /$ body weight $] \times 100$ and preference-saccharin $\mathrm{ml} /$ total $\mathrm{ml}$.
Male rats drank less avidly than did females during saccharin testing (Nance, Gorski, \& Panksepp, 1976; Valenstein, Kakolewski, \& Cox, 1967; Wade \& Zucker, 1969). Males also were more emotional in the open field. Thus, by these measures, male was to female as LoS was to HiS, as noted by Gray (1987) for the Maudsley Reactive and Nonreactive lines. Whether the line and sex differences have common mechanisms is currently under study in our laboratory.

\section{EXPERIMENT 2}

In Experiment 2, HiS and LoS rats were tested with solutions made with several different compounds. Although questions remain regarding the nature of the taste of saccharin for rats, it seems to have quinine-like ("bitter") and sucrose-like ("sweet") components (Dess, 1993). Thus, line differences in saccharin intake could occur because, relative to HiS rats, LoS rats are more sensitive to the bitter component, less sensitive to the sweet component, or both. If LoS rats are more sensitive to bitter, they should show suppressed intake of quinine solution. If they are less sensitive to sweet, they should drink less sugar solution. These possibilities were assessed using 24-h two-bottle tests with quinine and sucrose.

There also is a possibility that some other gustatory property of saccharin, or the emotional reaction to it, might account for the line difference. Consequently, additional animals were tested for ingestion of Polycose, a hydrolyzed starch. Polycose, which is consumed avidly by rats and is preferred to water, seems to have its own unique taste rather than tasting sugar-like or sweet (see Sclafani, 1991). If differences in saccharin intake are related to the general motivational properties of taste rather than to specific taste qualities, then the lines should differ on Polycose ingestion.

Solutions of Polycose and glucose alone also were compared with Polycose-saccharin and glucose-saccharin mixtures. Normally, adding saccharin to either Polycose or glucose enhances ingestion above the level seen with any of the three solutes alone (Sclafani, Einberg, \& Nissenbaum, 1987; Smith \& Foster, 1980). If, however, LoS rats are particularly sensitive to saccharin's bitter component, then their ingestion of both Polycose-saccharin and glucose-saccharin mixtures should be suppressed relative to that of HiS rats.

Many of these taste tests bear on the parallel between stress effects and differences between HiS and LoS lines. The quinine concentration was similar to that used to demonstrate stress-induced finickiness (Dess et al., 1989), and the sucrose solution is consumed more avidly after stress (Dess, 1992). Stress selectively reduces intake of a glucose-saccharin mixture when the alternative is a concentrated glucose solution (Dess \& Choe, 1994). If LoS rats are like rats exposed to inescapable tailshock, they should selectively reduce their intake of quinine- or saccharin-adulterated solutions and should consume more plain dilute sucrose than should HiS rats. If, on the other hand, line differences in saccharin ingestion reflect 
a global hedonic or motivational deficit in LoS rats, then LoS rats should consume less quinine, sucrose, glucose, and Polycose than should HiS rats, especially when any of these solutes is mixed with saccharin.

\section{Method}

Quinine and sucrose tests. The 60 rats from Experiment 1 were used for the quinine and sucrose tests. Saccharin testing in Experiment 1 was followed by two consecutive 24-h two-bottle (flavor vs. water) tests in the present experiment. The first test assessed ingestion of a $0.0002 \%$ quinine hydrochloride solution (Sigma Chemical, St. Louis, MO); the second test assessed ingestion of a $2 \%$ sucrose solution (ICN Biochemicals, Costa Mesa, CA). Both solutions were made with tap water. The left-right positions of flavor and water were balanced across rats and test days.

Polycose tests. Twenty naive HiS rats ( 6 males and 14 females) and 22 naive LoS rats ( 14 males and 8 females), 80-110 days of age, from two litters in Generations 5 and 6 of each line were reared and maintained as described for Experiment 1, except that weaning occurred at 21 days of age.

After baseline water-intake and body-weight determination, each rat was given two consecutive 24-h two-bottle Polycose versus water tests. In one test, $2 \%$ Polycose (Ross Laboratories, Columbus, $\mathrm{OH}$ ) solution was available in one bottle. In the other test, a mixture of $2 \%$ Polycose and $0.1 \%$ saccharin (Sigma Chemical, St. Louis, MO) was available in one bottle. Polycose solutions were made daily with tap water. All fluids were presented in glass bottles. The order of the tests and left-right positions of flavor and water were balanced for line and sex.

Glucose test. Thirty-seven HiS rats ( 11 males and 26 females) from six litters and $35 \mathrm{LoS}$ rats (11 males and 24 females) from five litters in Generations 5 and 6 of each line were reared and maintained as above. All rats were 80-110 days of age at the beginning of testing. Each rat received simultaneous access to a $24 \%$ glucose solution in one glass bottle (ICN Biochemicals, Costa Mesa, CA) and a mixture of $2 \%$ glucose and $0.1 \%$ saccharin in tap water in the other bottle (Sigma Chemical, St. Louis, MO).

Prior experience with various flavors among HiS and LoS rats were the same, but the histories of the males and females differed. Males had received three $24-\mathrm{h}$ two-bottle tests with $0.1 \%$ saccharin, and females had received separate 24 -h tests with $0.1 \%$ saccharin, $0.45 \%$ saline, and $0.9 \%$ saline as part of a pilot study.

\section{Results}

Important results from quinine, sucrose, Polycose, and glucose tests are shown in Figure 3. Preference scores are shown for quinine (quinine intake in milliliters/total fluid intake in milliliters). For sucrose and Polycose tests, avidity scores were calculated as described for Experiment 1 . For the simultaneous glucose test, consumption of each solution is expressed as a percentage of body weight without water baseline transformation.

Quinine and sucrose tests. Total fluid consumption during the quinine test did not differ between lines or sexes. However, quinine preference was lower for LoS rats than for HiS rats $[F(1,55)=5.73]$. The test of the bodyweight covariate showed that lighter rats tended to have lower quinine preference scores than did heavier rats of the same sex $[F(1,55)=3.00]$, as reported previously (Dess \& Chapman, 1990).

During sucrose testing, LoS rats drank less total fluid than did HiS rats, and males drank less than did females. The line difference was larger among females than among males. Sucrose preference, on the other hand, did not differ between lines or sexes (all mean preference scores $>0.95$ ). A two-factor ANOVA (line $\times$ sex) on avidity scores yielded significant main effects of line and sex $\left[F_{\mathrm{S}}(1,56)>17.3\right]$ and a significant line $\times$ sex interaction $[F(1,56)=6.20]$. A line $\times$ sex ANOVA on preference scores yielded no significant effects. Neither avidity nor preference covaried with body weight.

Polycose tests. LoS rats weighed more and drank more water during baseline than did HiS rats $[F \mathrm{~s}(1,38)=4.35$ and 5.27, respectively]. Preliminary ANCOVAs showed no covariation between either baseline measure and any test behavior.

Several rats unexpectedly emptied their $250-\mathrm{ml}$ bottle of Polycose. Bottle emptying was equally common for the two lines: $3 \mathrm{HiS}$ rats and $3 \mathrm{LoS}$ rats emptied their bottles of plain Polycose, and $6 \mathrm{HiS}$ rats and $7 \mathrm{LoS}$ rats emptied their bottles of the Polycose-saccharin mixture. Bottle emptiers were retested immediately with a $500-\mathrm{ml}$ bottle of the same solution, and the larger of the two values was used.

Avidity and preference scores were analyzed separately for the plain Polycose and the Polycose-saccharin solutions. Avidity for neither the Polycose solution nor

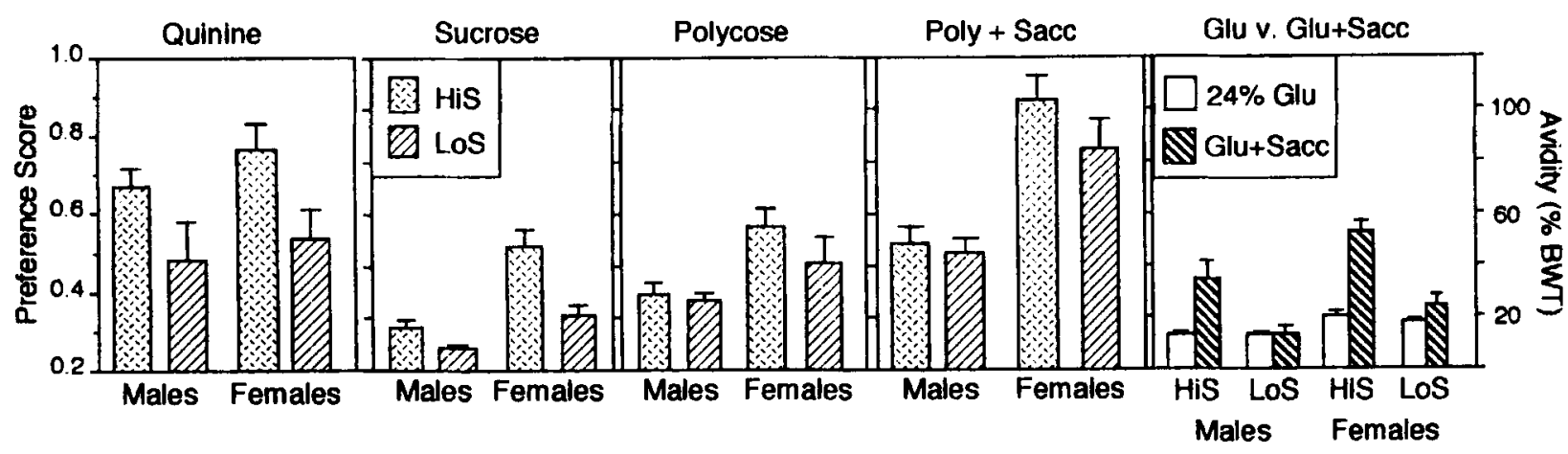

Figure 3. Experiment 2: The left panel shows preference for $0.0002 \%$ quinine hydrochloride, adjusted for baseline body weight. Avidity scores for $2 \%$ sucrose, $2 \%$ Polycose, and $2 \%$ Polycose $+0.1 \%$ saccharin show change in total fluid intake as a percentage of body weight. Avidity for two glucose solutions presented simultaneously (right panel) is expressed relative to body weight without water baseline transformation. 
the Polycose-saccharin mixture differed between lines (all $p s>.20)$. Preference scores for Polycose (not shown), either alone or in mixture with saccharin, were at least 0.92 in all groups. Females drank both the plain Polycose solution $[F(1,38)=8.26]$ and the Polycose-saccharin mixture $[F(1,38)=26.98]$ more avidly than did males.

Reanalysis using only the animals that did not empty their bottles led to the same conclusions. As in the intact groups, neither avidity nor preference differed significantly between lines.

Glucose test. Neither baseline water intake nor body weight differed between lines. LoS rats consumed as much of the plain, concentrated glucose solution as did HiS rats but consumed much less of the glucose-saccharin mixture. HiS rats showed the usual "polydipsic" response to the glucose-saccharin mixture.

Males' and females' data were analyzed separately because the sexes had different flavor histories and sample sizes differed considerably. A mixed-design ANOVA (line $\times$ solution) on the males' data yielded significant main effects of line $[F(1,20)=7.63]$ and solution $[F(1,20)$ $=6.95]$ and a significant line $\times$ solution interaction $[F(1,20)=6.77]$. Analysis of the interaction indicated that the lines differed on intake of the saccharin mixture $[F(1,20)=7.60]$ but not of the $24 \%$ glucose solution. A mixed-design ANOVA (line $\times$ solution) on females' data also yielded significant main effects of line $[F(1,48)=$ $27.06]$ and solution $[F(1,48)=38.25]$ and a significant line $\times$ solution interaction $[F(1,48)=16.36]$. Analysis of the interaction indicated that the lines differed on intake of the saccharin mixture $[F(1,48)=24.86]$ but again not of the $24 \%$ glucose.

\section{Discussion}

These tests with various tastants show that the difference between LoS and HiS lines is not unique to saccharin and that no single mechanism is responsible. Quinine and sucrose tests yielded differences between HiS and LoS lines on different intake measures. Quinine preference, but not avidity, distinguished the lines. For sucrose, on the other hand, the lines differed in avidity despite equally high preference scores, suggesting that detection threshold was not responsible for the differential avidity at this concentration. The specificity of these effects for prototypically bitter and sweet tastants implies that the preference measure is more sensitive to hedonically negative or aversive taste qualities, whereas the avidity measure is more sensitive to hedonically positive or appetitive taste qualities. These generalities seem to hold true at least at suprathreshold concentrations. As the LoS and HiS lines differed on both preference and avidity for saccharin, selection on the basis of saccharin intake seems to have acted on more than one taste-related mechanism.

Even a weak Polycose solution was highly preferred to water and consumed avidly by HiS and LoS rats alike. Moreover, the addition of saccharin to Polycose increased consumption in both lines to about the same degree. This result contrasts sharply with the line difference in the glucose test, in which adding saccharin to dilute glucose failed to increase its consumption relative to unadulterated glucose among LoS rats. Interpretation of the Polycose data is complicated by a possible ceiling effect, which might have masked line differences in avidity. Indeed, more recent (unpublished) data show greater avidity, but still not a stronger preference, for Polycose in the HiS line. Nonetheless, the present results for Polycose are informative. The high Polycose avidity of LoS rats demonstrates that their modest intake of saccharin, sucrose, and glucose-saccharin solutions is not due to intolerance for, or disinterest in, consuming a large volume of fluid. In addition, the similarity of the lines in the Polycose tests at this point in the breeding project-a point at which reliable differences for other flavors have appeared - suggests that selection has exerted more pressure on the processing of some flavors than of others. As such, any adequate account of the line differences must posit some specificity in the relationship between various taste qualities and ingestion.

The lower quinine preference and avidity for saccharin and saccharin-adulterated glucose solutions by LoS rats parallel effects of exposure to inescapable tailshock or restraint among randomly bred rats (Dess \& Choe, 1994; Plaznik et al., 1989). The parallel breaks down, however, for sucrose. Although tailshock or tailpinch enhances dilute sucrose intake (Bertiere, Mame Sy, Baigts, Mandenoff, \& Apfelbaum, 1984; Dess, 1992), LoS rats drank less avidly during the sucrose test. Thus, the symmetrical "finickiness" of inescapably shocked rats does not characterize LoS rats. Rather, with the notable exception of their responsiveness to saccharin adulteration of Polycose, LoS rats seem "anhedonic." Interestingly, chronic mild stress reduces sucrose intake (Katz, 1982; Muscat, Towell, \& Willner, 1988), so such regimes may provide a better experimental model of life as a LoS rat. Alternatively, the means by which stress affects sucrose intake may be unrelated to those yielding line differences. For example, line differences in sucrose intake might arise from variations in gustatory receptor densities, which are unlikely to be the sole mediator of stress effects. Only additional study can resolve the discrepancy.

These taste tests leave many questions unanswered. Single concentrations of tastants other than saccharin were chosen to allow comparison between these and prior studies. As a consequence, however, taste quality was confounded with intensity and, in some cases, caloric density. In addition, for all but the Polycose tests, rats had been given prior experience with saccharin to provide an uncontaminated basis on which to select animals for breeding. Unfortunately, saccharin pretesting raises the possibility that carry-forward effects played a role in the line differences observed for quinine, sucrose, and glucose solutions. To evaluate these possibilities, we are currently establishing concentration functions for all of these and other tastants without preexposure to saccharin. 


\section{EXPERIMENT 3}

Experiment 3 was undertaken to provide a more detailed analysis of the reaction to saccharin across a number of concentrations in order to further assess the role of aversive and appetitive taste properties in saccharin ingestion in the HiS and LoS lines. Rats were tested with five concentrations of saccharin ranging from $0.05 \%$ to $1.0 \%$. The concentration function in this range for rats is normally an inverted $U$ for both avidity and preference measures. An absolute preference (preference scores greater than 0.50 ) is maintained up to saccharin concentrations of $0.5 \%-0.7 \%$, with aversion (preference scores less than 0.50 ) appearing at higher concentrations. Aversive taste properties have been implicated in the descending limb of the concentration-intake function (see Dess, 1993). If the lines differ on saccharin ingestion because saccharin's intensity or appetitive properties are weaker in LoS rats, the lines' concentration functions should be similar with respect to shape and whether the functions fall above or below a preference score of 0.50 . If saccharin's aversive properties play a role, LoS rats should become averse to saccharin at a lower concentration than should HiS rats.

\section{Method}

Subjects and Apparatus. Twenty naive HiS rats ( 10 males and 10 females) from five litters and 29 naive LoS rats $(10$ males and 19 females) from four litters of Generation 8 were reared and maintained as were the Polycose- and glucose-tested subjects in Experiment 2. The rats were $80-110$ days of age at the beginning of the study. All testing occurred in the rats' home cages.

Procedure. After baseline water-intake and body-weight determination, each rat received two consecutive $24-\mathrm{h}$ two-bottle tests at each of five saccharin concentrations $(0.05 \%, 0.1 \%, 0.25 \%$, $0.7 \%$, and $1.0 \%$ ), first in ascending order and then in descending order. A water-only "day off" separated the ascending and descending series. Distilled water and glass bottles were used throughout. The left-right positions of saccharin and water were balanced across rats and test days.

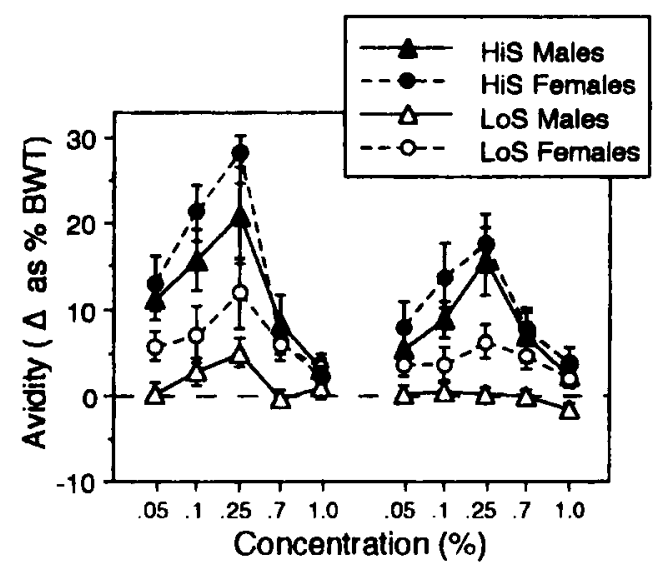

Run 1

Run 2

Figure 4. Experiment 3: Avidity for $0.05 \%-1.0 \%$ saccharin in two tests at each concentration. Concentrations were presented in ascending order in Run 1 and in descending order in Run 2.

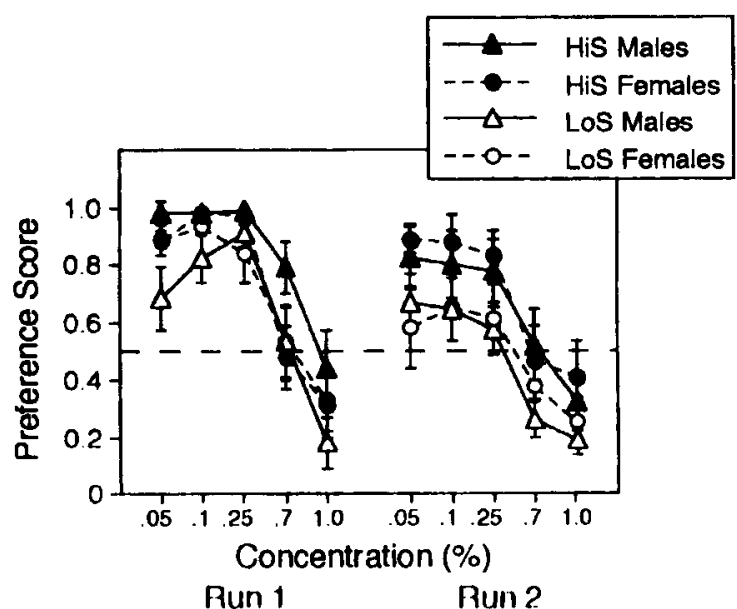

Figure 5. Saccharin preference in Experiment 3.

\section{Results and Discussion}

Neither baseline water intake nor body weight differed between lines. Avidity scores for both series are shown in Figure 4. The concentration-intake functions have the expected inverted-U shape, with some flattening in the second run. The LoS rats' functions are flatter than those of the HiS rats. At the highest concentration, no difference between lines or runs is apparent.

A mixed-design ANOVA (line $\times$ sex $\times$ run $\times$ concentration) yielded main effects of line $[F(1,35)=18.99]$, run $[F(1,35)=17.39]$, and concentration $[F(4,140)=$ $38.35]$ and significant line $\times$ concentration $[F(4,140)=$ 14.09] and run $\times$ concentration $[F(4,140)=6.80]$ interactions. Analysis of the line $\times$ concentration interaction indicated that the lines differed significantly at up to $0.7 \%[F \mathrm{~s}(1,35)=11.63,28.13,22.56$, and 5.12 , respectively], but not at $1.0 \%$.

Preference scores are shown in Figure 5. Saccharin preference declined as concentration approached $0.7 \%$ and decreased from the first to the second test series. As was the case for avidity, these trends were clearer for lower concentrations. LoS rats preferred saccharin less strongly than HiS rats at lower concentrations and were more averse than $\mathrm{HiS}$ rats to saccharin at the highest concentration. A mixed-design ANOVA yielded main effects of line $[F(1,35)=5.93]$, run $[F(1,35)=15.67]$, and concentration $[F(4,140)=66.70]$ and a significant run $\times$ concentration interaction $[F(4,140)=4.26]$.

Although some aspects of line differences in avidity and/or preference appeared to vary as a function of sex, no effects involving sex were significant.

\section{EXPERIMENT 4}

The preceding findings concerning saccharin, quinine, and glucose-saccharin solutions in LoS rats are reminiscent of the effects of inescapable shock on ingestion in the helplessness paradigm (Dess, 1992; Dess \& Chapman, 1990; Dess, Chapman, \& Minor, 1988). Moreover, 
the greater emotionality expressed by LoS rats in the open-field test of Experiment 1 also has been shown to be positively related to stress vulnerability in randomly bred rats (Minor et al., 1994) and selectively bred rats (Buda et al., 1994; Pucilowski, Overstreet, Rezvani, \& Janowsky, 1993). Except for the results from the dilute sucrose test, then, LoS rats behave much like stressed rats.

Experiment 4 provided an initial test for differences between LoS and HiS rats in the impact of an experimental stressor. Some male HiS and LoS rats were exposed to unsignaled inescapable tailshock, while other rats from each line remained in their home cages. Because severe inescapable shock parameters overwhelm individual differences in stress vulnerability (Minor et al., 1994), we used a smaller number of inescapable shocks $(v i z, 60)$ for the present study than is typical of our previous work on distress and helplessness in nonselected rats (viz, 100 or 120). These shock parameters were presumed to be potent enough to allow for the expression of any line difference in stress vulnerability without overwhelming them (Minor et al., 1994). Food, saccharin, and water intake and body weight were measured over the 3 days following stress treatments.

Experience with inescapable tailshock reduces food intake, saccharin avidity, and body weight in randomly bred male rats (Dess, 1992; Dess, Raizer, et al., 1988; Dess et al., 1989; Weiss, 1968). To the extent that the flavor preferences and fearfulness of LoS rats reflect processes that also are involved in stress vulnerability, shock treatment should have a larger impact on some (or all) of these measures in LoS rats.

\section{Method}

Subjects. Twenty-two HiS and 20 LoS male rats (90-110 days of age) from six litters in each lines' fifth generation were reared and maintained as described for Experiment 3.

Apparatus. All measures were taken in the home cages. Tap water and $0.1 \%$ saccharin (Sigma Chemical, St. Louis, MO) solution were presented in glass bottles with stainless steel spouts and rubber stoppers.

The stress session was administered in individual $14 \times 11 \times$ $17 \mathrm{~cm}$ clear acrylic restraining tubes. A rat's tail extended through the end of the tube and was taped to an acrylic rod with adhesive tape. Two electrodes, coated with electrode paste, were taped to the tail $1.5 \mathrm{~cm}$ apart. Shock was delivered to the electrodes from constant-current shock generators (Lafayette Instruments Model 82400 , Lafayette, IN). The chambers were located in illuminated white sound-attenuating chests. Ventilation fans mounted behind the rear wall provided masking noise. Experimental events were computer controlled.

Procedure. Food and water intake were measured over a 48-h period. Prestress body weight was measured on the second baseline day, after which rats in each line were assigned to either the shock or the no-shock condition, matched for body weight (HiS, $n=11$ in each condition; $\operatorname{LoS}, n=10$ in each condition). The noshock groups remained in their home cages. The stress session began for the shock groups at approximately 9:00 a.m. PST and consisted of exposure to 60 (5-sec, $0.6-\mathrm{mA})$ tailshocks on a variable-time $60-\mathrm{sec}$ schedule (range: $20-230 \mathrm{sec}$ ). When these tats were returned to their home cages, all rats received a $250-\mathrm{ml}$ bottle of saccharin solution and a $100-\mathrm{ml}$ bottle of water. Chow, saccharin, and water intake and body weight were measured daily
Table 2

Experiment 3: Prestress Body Weight and Average Daily Food and Water Intake in HiS and LoS Male Rats

\begin{tabular}{lccccc}
\hline & \multicolumn{2}{c}{ HiS } & Rats & & \multicolumn{2}{c}{ LoS Rats } \\
\cline { 2 - 3 } \cline { 5 - 6 } & $M$ & SEM & & \multicolumn{1}{c}{$M$} & SEM \\
\hline Body weight (grams) & 456.6 & 14.6 & & 447.2 & 9.7 \\
Food intake (\% body weight) & 6.8 & 0.2 & & 6.3 & 0.2 \\
Water intake (\% body weight) & 12.6 & 0.5 & & 10.7 & 0.6 \\
\hline
\end{tabular}

for 3 days after the stress session. The left-right positions of saccharin and water were balanced across rats and test days.

\section{Results and Discussion}

Baseline measures. Baseline body weight and food and water intake (relative to body weight) are shown in Table 2. Body weight and food intake did not differ between the lines. However, HiS rats drank more water than did $\operatorname{LoS}$ rats $[F(1,40)=5.87]$. When baseline water intake was used as a covariate in analysis of test data, it covaried positively with saccharin avidity $[F(1,37)=$ $9.50]$ and with weight gain after stress $[F(1,37)=19.32]$. Therefore, ANCOVA results are reported for those measures.

Poststress measures. Food intake after stress is shown as change from prestress baseline in Figure 6. Stress initially reduced food intake among LoS rats but not among HiS rats. A mixed-design ANOVA (line $X$ stress condition $\times$ day) yielded main effects of stress $[F(1,38)=$ $6.76]$ and day $[F(2,76)=14.09]$ and a significant day $\times$ stress interaction $[F(2,76)=10.32]$. Most importantly, the line $\times$ stress interaction also was significant $[F(1,38)=$ 7.00]. Analysis of this interaction with separate ANOVAs yielded a stress effect $[F(1,18)=10.66]$ and a day $\times$ stress interaction $[F(2,36)=7.37]$ only for LoS rats.

Avidity and preference for saccharin in each group over the 3 test days are presented in Figure 7. Stressed rats drank less avidly than did nonstressed controls $[F(1,37)=$

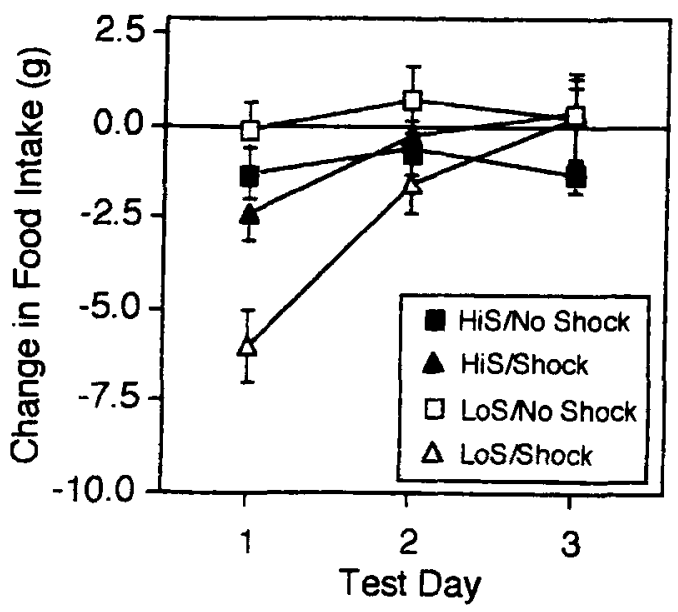

Figure 6. Experiment 4: Food intake on 3 test days, expressed as change from baseline daily food intake, among male HiS and LoS rats, half of whom had been exposed to inescapable tailshock. 


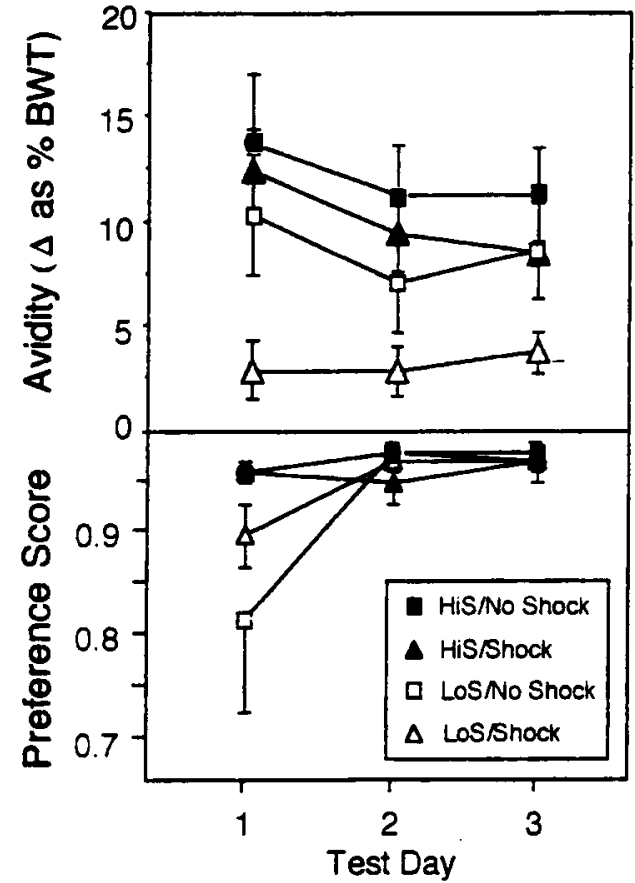

Figure 7. Experiment 4: Saccharin avidity and preference during testing.

$4.51]$ and avidity declined over days $[F(2,76)=4.78]$. LoS rats drank less avidly throughout testing $[F(1,37)=$ 5.15] and initially preferred saccharin less strongly to water than did HiS rats [day $\times$ line interaction; $F(2,76)=$ 4.78]. Although the initial reduction of saccharin avidity by stress was numerically larger among LoS rats, the line $X$ stress interaction was not significant.

Body weight (not shown) generally increased during testing $[F(2,76)=43.42]$, and did so more in the absence of stress $[F(1,37)=4.09]$. Body weight was not affected differentially by stress in the two lines, despite the reduced eating and drinking among stressed LoS rats.

This experiment provides limited support for the idea that LoS rats are more vulnerable to stress than HiS rats. Stress reduced food intake only among the former. That the differential effect of stress on food intake was not accompanied by line differences in weight loss suggests a difference in how the two lines respond metabolically or viscerally to stress. Direct tests of the responses of LoS and HiS rats to metabolic challenges are under way.

Although the data hint at a differential effect of stress on saccharin avidity, the effect was not reliable. Whether more robust line differences in stress-induced changes in flavor intake might be obtained with other stressor or test parameters remains to be determined.

\section{GENERAL DISCUSSION}

We have successfully bred for individual differences in the behavior of rats toward saccharin, as expected (Nachman, 1959). Selection on the basis of volume of saccharin solution consumed has yielded differences in both avidity and preference for saccharin and saccharinadulterated glucose solutions. Effects for other tastants were more specific. The lines differed only on avidity for dilute sucrose solution and only on preference for quinine. Neither avidity nor preference differed between lines for Polycose, the taste of which appears to be mediated by distinct gustatory receptors (Sclafani, 1991). Thus, selective breeding on the basis of saccharin intake appears to have yielded earlier line differences for some tastes than for others.

Only two litters per line were used in some comparisons (in Experiment 1 and in the quinine, sucrose, and Polycose tests in Experiment 2), potentially raising the concern that the results might have been spurious or peculiar to those litters (Blizard, 1992). Several findings, however, should allay such concern. First, litter-by-litter inspection of the data suggested that each litter contributed about equally to line differences or, in the case of Polycose and $24 \%$ glucose, the absence thereof. Second, in a 24-h two-bottle saccharin test conducted after Polycose testing in Experiment 3, the HiS rats did drink more than the LoS rats $[F(1,38)=5.57]$. Thus, although only two litters in each line were used, the expected line difference in saccharin intake was observed in those litters. Finally, all comparisons of the lines subsequent to the Polycose tests involved at least four litters from each line. It seems unlikely, then, that conclusions regarding line differences were idiosyncratic to the litters used in any particular part of the study.

The role of genetics in these line differences is unclear. The unusual rats used to begin the lines were males and therefore presumably contributed only their genes. This circumstance implies some genetic involvement, especially given the clear role of genetics in bitter and sweet taste sensitivity in humans (Bartoshuk, 1979; Bartoshuk, Rifkin, Marks, \& Hooper, 1988; Capeless, Whitney, \& Azen, 1992; Gent \& Bartoshuk, 1983; Looy \& Weingarten, 1992) and mice (Belknap et al., 1992; Lush, 1989; Pelz, Whitney, \& Smith, 1973). However, genetics could play a tiny role that is elaborated and overlaid by more potent congenital and postnatal environmental influences (Blizard, 1992). A related, unresolved question of interest to behavior geneticists concerns the direction(s) in which we have shifted saccharin intake relative to the stock population. Initial tests with laboratoryreared, randomly bred Holtzman rats suggest that selection first reduced saccharin ingestion in the LoS line but has gradually increased saccharin ingestion in the HiS line as well, such that both lines differ from the stock population. Determination of whether any genetic bases of the line differences are linked and of how their expression is modified by experience will require techniques (e.g., replicate lines; cross-fostering) not yet undertaken.

Although the parallel is not perfect, the original hypothesis that individual differences in taste, emotionality, and stress vulnerability have some common basis is encouraged by these data. Related work with humans 
also points to a link between affect and bitter taste (Dess \& Chapman, 1990; Mascie-Taylor, McManus, MacLarnon, \& Lanigan, 1983; Whittemore, 1986) and between stress, temperament, and saccharin's perceived sweetness and bitterness (Dess \& Edelheit, 1994). Unraveling the specific relationships between taste quality and affective processes will require substantial further work.

\section{REFERENCES}

BartoshuK, L. M. (1979). Bitter taste of saccharin related to the genetic ability to taste the bitter substance 6- $n$-propylthiouracil. Science, 205, 934-935.

Bartoshuk, L. M., Rifkin, B., Marks, L. E., \& Hooper, J. E. (1988). Bitterness of $\mathrm{KCl}$ and benzoate: Related to genetic status for sensitivity to PTC/PROP. Chemical Senses, 13, 517-528.

Belknap, J. K., Crabie, J. C., Plomin, R., McClearn, G. E., Sampson, K. E., O'ToOle, L. A., \& Gora-Maslak, G. (1992). Singlelocus control of saccharin intake in $\mathrm{BXD} / \mathrm{Ty}$ recombinant inbred (RI) mice: Some methodological implications for RI strain analysis. Behavior Genetics, 22, 81-100.

BERLYNE, D. E. (1969). The reward value of indifferent stimulation. In J. T. Tapp (Ed.), Reinforcement and behavior (pp. 178-214). New York: Academic Press.

Bertiere, M. C., Mame Sy, T., Baigts, F., Mandenoff, A., \& ApfelBAUM, M. (1984). Stress and sucrose hyperphagia: Role of endogenous opiates. Pharmacology, Biochemistry \& Behavior, 20, 675-679.

BLIZARD, D. A. (1992). Recombinant-inbred strains: General methodological considerations relevant to the study of complex characters. Behavior Genetics, 22, 621-633.

Brush, F. R., Del Paine, S. N., Pellegrino, L. J., Rykaszewski, I. M., Dess, N. K., \& Collins, P. Y. (1988). CER suppression, passiveavoidance learning, and stress-induced suppression of drinking in the Syracuse high- and low-avoidance strains of rats (Rattus norvegicus). Journal of Comparative Psychology, 102, 337-349.

Buda, M., Lachuer, J., Devauges, V., Barbabli, B., Blizard, D., \& SARA, S. J. (1994). Central noradrenergic reactivity to stress in Maudsley rat strains. Neuroscience Letters, 167, 33-36.

Capeless, C. G., Whitney, G., \& Azen, E. A. (1992). Chromosome mapping of Soa, a gene influencing gustatory sensitivity to sucrose octaacetate in mice. Behavior Genetics, 22, 655-663.

DESs, N. K. (1991). Ingestion and emotional health. Human Nature, 2, 235-269.

DESs, N. K. (1992). Divergent responses to saccharin and sucrose availability after stress in rats. Physiology \& Behavior, 52, 115-125.

DEss, N. K. (1993). Saccharin's aversive taste in rats: Evidence and implications. Neuroscience \& Biobehavioral Reviews, 17, 359-372.

DESS, N. K., \& ChAPMAN, C. D. (1990). Individual differences in taste, body weight, and depression in the "helplessness" rat model and in humans. Brain Research Bulletin, 24, 669-676.

Dess, N. K., Chapman, C. D., \& Minor, T. R. (1988). Inescapable shock increases finickiness about drinking quinine-adulterated water in rats. Learning \& Motivation, 19, 408-422.

Dess, N. K., \& ChOE, S. (1994). Stress selectively reduces sugar+ saccharin mixture intake but increases proportion of calories consumed as sugar by rats. Psychobiology, 22, 77-84.

Dess, N. K., \& Edelheit, D. (1994). Stress and temperament predict saccharin's taste in humans. Unpublished manuscript.

Dess, N. K., Minor, T. R., \& BREWER, J. (1989). Suppression of feeding and body weight by inescapable shock: Modulation by quinine adulteration, stress reinstatement, and controllability. Physiology \& Behavior, 45, 975-983.

Dess, N. K., Raizer, J., Chapman, C., \& Garcia, J. (1988). Stressors in the learned helplessness paradigm: Effects on body weight and conditioned taste aversion in rats. Physiology \& Behavior, 44, 483-490.

Dess, N. K., \& VANDERWEELE, D. A. (1994). Lithium chloride and inescapable, unsignaled tail shock differentially affect meal patterns of rats. Physiology \& Behavior, 56, 203-207.
Gent, J. F., \& BartoshuK, L. M. (1983). Sweetness of sucrose, neohesperidin dihydrochalcone, and saccharin is related to genetic ability to taste the bitter substance 6- $n$-propylthiouracil. Chemical Senses, 7, 265-272.

Gosnell, B. A., \& KraHN, D. D. (1992). The relationship between saccharin and alcohol intake in rats. Alcohol, 9, 203-206.

GRAY, J. (1987). The psychology of fear and stress (pp. 92-114). Cambridge: Cambridge University Press.

Helmstetter, F. J., \& Fanselow, M. S. (1993). Aversively motivated changes in meal patterns of rats in a closed economy: The effects of shock density. Animal Learning \& Behavior, 21, 168-175.

JACOBS, H., \& Sharma, K. (1969). Taste versus calories: Sensory and metabolic signals in the control of food intake. Annals of the New York Academy of Sciences, 157, 1084-1125.

KATZ, R. J. (1982). Animal model of depression: Pharmacological sensitivity of a hedonic deficit. Pharmacology, Biochemistry \& Behavior, 16, 965-968.

Looy, H., \& Weingarten, H. P. (1992). Facial expressions and genetic sensitivity to 6-n-propylthiouracil predict hedonic response to sweet. Physiology \& Behavior, 52, 75-82.

LusH, I. E. (1989). The genetics of tasting in mice: VI. Saccharin, acesulfame, dulcin and sucrose. Genetics Research, 53, 95-99.

Mascie-Taylor, C. G. N., McManus, I. C., Maclarnon, A. M., \& LANIGAN, P. M. (1983). The association between phenylthiocarbamide (PTC) tasting ability and psychometric variables. Behavior Genetics, 13, 191-196.

Minor, T. R., Dess, N. K., Ben-David, E., \& Chang, W. C. (1994). Individual differences in neophobia and vulnerability to inescapable shock. Journal of Experimental Psychology: General, 20, 402-412.

Muscat, R., Towell, A., \& Willner, P. (1988). Changes in dopamine autoreceptor sensitivity in an animal model of depression. Psychopharmacology, 94, 545-550.

NaChMAN, M. (1959). The inheritance of saccharin preference. Journal of Comparative \& Physiological Psychology, 52, 451-457.

NANCE, D., Gorski, R., \& PANKSEPP, J. (1976). Neural and hormonal determinants of sex differences in food intake and body weight. In D. Novin, W. Wyrwicka, \& G. Bray (Eds.), Hunger: Basic mechanisms and clinical implications (pp. 257-271). New York: Raven Press.

Overstreet, D. H., Kampov-Polevoy, A. B., Rezvani, A. H., MurRelle, L., Halikas, J. A., \& Janowsky, D. S. (1993). Saccharin intake predicts ethanol intake in genetically heterogeneous rats as well as different rat strains. Alcoholism: Clinical \& Experimental Research, 17, 366-369.

Pelz, W., Whitney, G., \& Smith, J. (1973). Genetic influences on saccharin preference of mice. Physiology \& Behavior, 10, 263-265.

Plaznik, A., Stefanski, R., \& Kostowski, W. (1989). Restraint stress-induced changes in saccharin preference: The effect of antidepressive treatment and diazepam. Pharmacology, Biochemistry \& Behavior, 33, 755-759.

Pucilowski, O., Overstreet, D. H., Rezvani, A. H., \& Janowsky, D. S. (1993). Chronic mild stress-induced anhedonia: Greater effect in a genetic rat model of depression. Physiology \& Behavior, 54, 1215-1220.

ROYCE, J. R. (1977). On the construct validity of open-field measures. Psychological Bulletin, 84, 1098-1106.

SCLAFANI, A. (1991). The hedonics of sugar and starch. In R. C. Bolles (Ed.), The hedonics of taste (pp. 59-87). Hillsdale, NJ: Erlbaum.

Sclafani, A., Einberg, L. T., \& Nissenbaum, J. W. (1987). Influence of saccharin on Polycose, sucrose, and glucose intake and preference in rats. Neuroscience \& Biobehavioral Reviews, 11, 223-229.

SCAlfani, A., SPringer, D., \& Kluge, L. (1976). Effects of quinine adulterated diets on the food intake and body weight of obese and non-obese hypothalamic rats. Physiology \& Behavior, 16, 631-640.

Sinclair, J. D., Kampov-Polevoy, A., StEwarT, R., Li, T.-K. (1992). Taste preferences in rat lines selected for low and high alcohol consumption. Alcohol, 9, 155-160.

SMITH, J. C., \& FosTER, D. F. (1980). Some determinants of intake of glucose+saccharin solutions. Physiology \& Behavior, 25, 127-133.

Valenstein, E., Kakolewski, J., \& Cox, V. (1967). Sex differences in taste preference for glucose and saccharin solution. Science, 156, 942-943. 
Vaswani, K., Tejwani, G. A., \& Mousa, S. (1983). Stress induced differential intake of various diets and water by rat: The role of the opiate system. Life Sciences, 12, 1983-1996.

WADE, G. N., \& ZUCKER, I. (1969). Hormonal and developmental influences on rat saccharin preferences. Journal of Comparative \& Physiological Psychology, 69, 291-300.

WaLSH, R. N., \& Cummins, R. A. (1976). The open-field test: A critical review. Psychological Bulletin, 83, 482-504.
WeISs, J. M. (1968). Effects of coping responses on stress. Journal of Comparative \& Physiological Psychology, 65, 251-260.

WhitTeMORE, P. B. (1986). Phenylthiocarbamide (PTC) tasting and reported depression. Journal of Clinical Psychology, 42, 260-263.

Willner, P., Towell, A., Sampson, D., Sophokleous, S., \& Muscat, R. (1987). Reduction of sucrose preference by chronic unpredictable mild stress, and its restoration by a tricyclic antidepressant. Psychopharmacology, 93, 358-364.

(Manuscript received June 29, 1994;

revision accepted for publication March 7, 1995.) 